\title{
Uso de redes sociales en el desarrollo de estrategias de lectura crítica hipertextual en estudiantes universitarios
}

\section{Use of Social Networks and Hypertext Critical Reading Strategies in University Students}

\author{
Doris Elida Fuster-Guillén* \\ Universidad Nacional Mayor de San Marcos, Lima, Perú \\ ORCID: https://orcid.org/0000-0002-7889-2243 \\ Arthur Serrato-Cherres \\ Universidad César Vallejo, Lima, Perú \\ ORCID: https://orcid.org/0000-0003-3525-6607 \\ Raúl Gonzales Álvarez \\ Universidad Nacional Daniel Alcides Carrión, Cerro De Pasco, Perú \\ ORCID: https://orcid.org/0000-0001-6442-2534 \\ Nélida Flor Goicochea Euribe \\ Universidad Nacional Daniel Alcides Carrión, Cerro De Pasco, Perú \\ ORCID: https://orcid.org/0000-0002-7338-6085 \\ Patricia Edith Guillén Aparicio \\ Universidad de San Martín de Porres, Lima, Perú \\ ORCID: https://orcid.org/0000-0002-8143-3646
}

Recibido 16-07-19 Revisado 23-09-19 Aprobado 12-12-19 En línea 07-01-20

*Correspondencia

Email: dfusterg@unmsm.edu.pe
Citar como:

Fuster-Gullén, D., Serrato-Cherres, A., Gonzales, R., Goicochea, N., \& Guillén, P. (2020). Uso de redes sociales en el desarrollo de estrategias de lectura crítica hipertextual en estudiantes universitarios. Propósitos y Representaciones, 8(1), e432. doi: http://dx.doi.org/10.20511/pyr2020.v8n1.432 


\section{Resumen}

El objetivo del presente estudio fue determinar si el uso de redes sociales permite el manejo de estrategias de lectura crítica hipertextual en los estudiantes del primer año de la Escuela de Economía de la Universidad Nacional Federico Villareal (Perú), esto debido a que en la actualidad los estudiantes utilizan herramientas tecnológicas y las redes sociales (RRSS) para el desarrollo de sus actividades académicas. Asimismo, utilizan estrategias de lectura crítica hipertextual, entendidas estas como una combinación de textos, sonidos e imágenes que resulta un texto, lo que genera una lectura multisecuencial y, de ser el caso, también multilineal. El método de investigación que se aplicó fue el hipotético deductivo, bajo un diseño no experimental, transversal, descriptivo de enfoque cuantitativo y paradigma positivista. La población de estudio estuvo conformada por 150 estudiantes universitarios a quienes se les administró cuestionarios de uso de redes sociales y estrategias de lectura hipertextual. Ambos instrumentos fueron validados por juicio de expertos, cuya fiabilidad obtenida por alpha de cronbach fue de 0.801 y 0.918 respectivamente. El resultado que se obtuvo fue que el uso de redes sociales permite el manejo de las estrategias de lectura crítica hipertextual; asimismo desarrolla los elementos de pensamiento y la comprobación de razonamiento crítico.

Palabras clave: Redes sociales; Lectura crítica; Lectura hipertextual; Estrategias de lectura; Universidad.

\section{Summary}

The objective of the present study was to determine if the use of social networks allows the management of hypertextual critical reading strategies in the first year students of the School of Economics of the Federico Villareal National University (Peru), due to the fact that at present Students use technological tools and social networks (RRSS) for the development of their academic activities. Likewise, they use hypertextual critical reading strategies, understood as a combination of texts, sounds and images that results in a text, which generates a multisequential reading and, if necessary, also multilinear. The research method that was applied was the hypothetical deductive, under a non-experimental, transversal design, descriptive of quantitative approach and positivist paradigm. The study population was made up of 150 university students who were administered questionnaires on the use of social networks and hypertextual reading strategies. Both instruments were validated by expert judgment, whose reliability obtained by cronbach's alpha was 0.801 and 0.918 respectively. The result was that the use of social networks allows the management of hypertextual critical reading strategies; It also develops the elements of thought and the verification of critical reasoning.

Keywords: Social networks; Critical reading; Hypertextual reading; Reading strategies; College.

\section{Introducción}

Las redes sociales son un espacio que permite opinar, compartir información y crear contenidos en una comunidad virtual mediante la Web 2.0, como lo son Facebook, MySpace, Linkedin, Twitter, Youtube, entre otros. Estos espacios virtuales han atraído, desde su aparición, a millones de usuarios en el mundo. Es así que su uso está llevando a que muchos jóvenes en el mundo se alejen de la comunicación vía llamada telefónica por móvil o la comunicación personal conocida como face to face, para pasar a una comunicación textual, como es el caso de la mensajería, el cual también se ha convertido en un método muy apreciado en lo que se refiere a comunicación sincrónica (Schwarz, 2011). Asimismo, afirman Almansa, Fonseca y Castillo (2013) los jóvenes, en su mayoría de 12 a 15 años, utilizan el aplicativo Facebook (FB), no solo para interactuar con sus amistades, sino como un medio de comunicación y socialización en general. De igual manera, resalta el estudio de San Martín y Mujica (2010) quienes refieren que los jóvenes usan extensivamente las plataformas en la web para formar redes sociales (RR SS). En esta misma línea destaca el aporte de Ruiz y Escurra 
(2013) quienes determinaron que, si bien existen altos niveles de uso de redes sociales, existen también niveles bajos en el desarrollo de la conciencia metacognitiva, así como en la utilización de estrategias metacognitivas en las lecturas. Otro estudio que amerita resaltar es el de Orellana (2012) quien precisa que los estudiantes utilizan el internet para informarse y estar informados, así como para obtener información en el ámbito académico. Como se puede observar, cada una de estas investigaciones concluyen en que el uso de redes sociales es inevitable, es por ello que es preciso que las universidades y los docentes adopten estrategias en las múltiples asignaturas para hacer de estas redes sociales una fortaleza en el logro académico y, sobre todo, para el desarrollo de capacidades y aprendizajes de los estudiantes.

La educación moderna se vale de la cultura digital, prueba de ello es que los estudiantes que hacen el uso de celulares, tablets o iPad, así como de diferentes dispositivos electrónicos (no solo para relacionarse con los demás, sino para ver en Internet diversos sucesos) graban las clases o toman fotografías de los apuntes, figuras, organizadores, etc. para compartirlos inmediatamente en la cuenta de Facebook o WhatsApp que previamente generaron como un solo grupo. El trabajo en equipo también se ha modificado al punto de que ya no es necesario que los integrantes se reúnan en un espacio físico para programar y desarrollar sus actividades, basta con "enlazarse" o "conectarse" desde cualquier lugar para hacer los trabajos, ayudándose de enlaces que incluyen en sus exposiciones mediante hipervínculos que llevan a diversas páginas web. Asimismo, cuando el docente les pide investigar sobre un tema, de inmediato utilizan el motor de búsqueda de Google. Es así que los estudiantes con la versión 2.0 viven utilizando la tecnología con acceso al Internet (cultura digital), también consumen, interactúan, producen y comparten la información, convirtiéndose el internet en una eficiente red de información enlazada en diversas presentaciones hipertextuales, tal es así que Galindo (2015) dio a conocer que los estudiantes hacen uso de las estrategias de lectura hipertextual, básicamente en lo que refiere a práctica de habilidades con orden superior, las cuales son la evaluación, la síntesis, el análisis, estos mostraron mejores resultados (puntajes) frente a lo que se refiere recabar información, así como comprensión y de aplicación, el cual mostraron bajos niveles , asimismo refiere que a partir de las particularidades del contexto donde se desenvuelven actualmente los estudiantes universitarios, es posible e inminente realizar el desarrollo de competencias digitales y mediales con una postura crítica, así como reflexiva ante lo que hallan y leen en la web. Por su parte Arancibia (2010) refiere que los razonamientos inferenciales frente al hipertexto son iguales al procesamiento de información adquiridos de la lectura de escritos, asimismo, se comprobó que la comprensión bajó, la modalidad discursiva hipertextual prima la participación activa. A continuación, se hace necesario adentrarnos al conocimiento teórico del uso de redes sociales y las estrategias hipertextuales que sustentan al presente trabajo.

\section{Uso de redes sociales}

Actualmente se cuenta con muchos conceptos, teorías sobre lo que se corresponde a redes sociales, sin embargo, no se cuenta con un acuerdo sobre ello. Cabe señalar, que la mayoría de los estudios coinciden que una red social es "un sitio, lugar, ubicación en la red que tiene como fin acceder al consumidor (usuario), comunicarse, relacionarse, trasmitir alguna información, así como también en la creación de grupos (comunidades), también se considera como una instrumento de "democratización de la información o datos que transforma o convierte a los usuarios en productores y receptores de contenidos".

Ahora bien, en el país de España, INTECO (Instituto Nacional de Tecnologías de la Comunicación) definió a las redes sociales como: "los servicios prestados a través de la web (internet) que admiten a los consumidores elaborar un perfil público el cual muestra no solo los datos personales si no también información del mismo usuario, disponiendo el consumidor de instrumentos que le permitan interactuar con diversos consumidores ya sean relacionados (también afines) o no a lo que se refiere el perfil mostrado públicamente" (p.19)

Existen diversos usos que se le da a las RRSS, sin embargo, se puede destacar cuatro empleos que fomentan el uso y en algunos casos el abuso; es de aclarar que son varios los perfiles que generan 
o muestran los usuarios en cuanto a su nivel cultural, sociológico, geográfico etc. Así mismo, debemos indicar que los usuarios tienen la necesidad de interactuar o comunicarse con otra gran masa de usuarios, por tanto, tienen la necesidad de estar unidos en relación a los cuatro usos o fines, tal como lo indica San Martín (2010, p.3): En el mantenimiento de amistades: mantener contacto o comunicación con sus amigos, familiares, compañeros, colegas, conocidos o ex compañeros de trabajo, etc., puesto que de no ser por las redes sociales se perdería la comunicación, casos que suceden en la vida real; en la creación de amistades: de los conocidos que tiene cada contacto, al usuario le sirve de base para tener nuevos contactos, amigos futuros y con ello lograr una cadena de creación de amistades cada vez que interactúen y conozcan; asimismo en el entretenimiento: en algunos casos además de las dos actividades anteriores se realizan paralelamente la exploración de los datos de otros usuarios, así como la actualización de un perfil o de informarse sobre la vida cotidiana de otras personas por último en la gestión interna de organizaciones empresariales: este punto corresponde o es utilizado por empresas, el cual consiste en crear una red social privada para realizar trámites, informes, video conferencias, comunicados o en su defecto crear la red privada para estar en contacto con profesionales del entorno o del sector. (p. 3).

\section{Lectura hipertextual}

Lo que se refiere a hipertexto, según Galindo (2015) está referido: "Es un texto ampliado en su definición etimológica directa es un texto enorme de creación transtextual, mezclado con sonidos e imágenes, en otras palabras, un texto líquido, que conlleva a una lectura no secuencial, multisecuencial o multilineal. Dichas características obligan al lector a tener una lectura dinámica, en otras palabras, se convierte en una especie de artesanos, una persona que zurce hilvana, recompone y ensambla piezas desordenadas, de diversos orígenes, manteniendo así en su cerebro el modelo resultante del lugar textual que se ofrece a la lectura. Utilizar al hipertexto, como su construcción, su lectura, nos obliga a realizar diferencias entre los tipos de hipertexto propuestos por Eco (2003, el hipertexto textual, es decir, el texto (literario) impreso que refiere de dos textos; el texto hipertextual, el cual significa que el texto que incluye nexos de hipervínculos para su despliegue, o, texto (no manuscritos o impresos sino digitalizados) que da acceso a los textos u obras que son sus referentes, y finalmente el hipertexto sistemático, el cual significa que el texto de múltiple formato que conforma parte de un conjunto de infinitas posibilidades, con repercusiones considerables en los requerimientos de habilidades de lectura y escritura hipertextual.

Este último tipo de hipertexto, el cual es el que llama la atención a los estudiantes y, por lo tanto, es el más importante para el presente trabajo. Este tipo de hipertexto presenta una diversidad de canales de información y comunicación el cual comporta un proceso de lectura multimodal, este hipertexto también obliga a realizar un zapping de lectura, de un formato a otro. De una página a otra. Es una realidad que requiere el desarrollo de una buena competencia lectora multimodal, "porque las nuevas textualidades pueden presentarse en soportes distintos a los convencionales que había utilizado el discurso verbal. En el marco de la denominada sociedad del conocimiento y de la comunicación, todos estamos en constante actividad lectora/receptora" (Mendoza, 2012, p. 80).

La web 2.0, quiere decir la web de la interactividad, genera que la lectura del hipertexto se convierta en una actividad dinámica, en donde el lector como productor reconstruye la información y genera una nueva. En esa línea Romea (2012) nos dice que: los hipervínculos o anclajes de determinadas palabras o frases que contiene un texto, con otras de otros textos, nos permiten seguir una lectura no lineal y, por tanto, añadir o eliminar información, así como fraccionamiento, reordenarla y asociarla a otras nuevas, etc. Incentiva o incita a los lectores a deambular por el documento de acuerdo a su interés, a través de los enlaces de navegación, en una acción eminentemente interactiva el cual invita a cada usuario a seleccionar los temas de un modo subjetivo y conforme con una o varias intencionalidades predeterminadas.

Teniendo como premisa que los estudiantes utilizan las redes sociales (RRSS) y otros equipos (herramientas) de lectura con la finalidad de captar y procesar la información, así como seleccionar cual es la más adecuada, es necesario que estos sepan manejar correctamente una lectura 
hipertextual el cual, con la herramienta indicada, mejoraría el desarrollo de su aprendizaje y formación profesional. Bajo ese contexto es recurrente ver en las aulas universitarias de los primeros ciclos, que muchos estudiantes presentan trabajos con información poco importante o desvinculada al propósito de la tarea, ello hace pensar que el hecho de que sean nativos digitales y, por ende, sepan usar con frecuencia recursos de carácter hipertextual en su vida cotidiana, hagan que desarrollen estrategias que los ayuden a leer y a seleccionar la información óptima en el internet para el desarrollo de sus trabajos o para ampliar su aprendizaje en la universidad.

Es por ello que el presente estudio parte de que la realidad (al menos la peruana) muestra que la gran cantidad de información que se obtiene en la red, se representa mediante el hipertexto, este último cambia las reglas de distribuir, almacenar y mostrar la información. Dicha información que se presenta es interactiva y de conectividad, desarrollando así hábitos cognitivos, siendo la corriente que defiende esta postura el Conectivismo, puesto que consideran que las herramientas que usamos definen y dan forma a nuestro pensamiento (Siemens, 2006).

Es en este contexto que se hace necesaria una disciplina de diseño hipertextual para crear entornos enfocados a facilitar la flexibilidad cognitiva, así como una capacidad cognitiva; por ello, la categoría de no-linealidad se adapte a la categoría de aprendizaje y también a las habilidades metacognitivas de los estudiantes, lo que implica un trabajo de diseño hipertextual personalizado o ajustado a grupos con determinadas características cognitivas. Partiendo de los puntos de vista antes indicados, se puede asumir que los estudiantes actuales, por encontrase en constante contacto con redes sociales y otras herramientas de carácter hipertextual, van creando formas distintas de leer y de procesar la información útil de la no útil, por ello se hace necesario saber si el uso de estos está asociado con estrategias críticas para la lectura de hipertextos, que cooperen a seleccionar y brindar valor a los distintos materiales que ellos utilizarán para desarrollar sus aprendizajes.

Es común que algunos padres de familia y maestros perciban que los estudiantes universitarios que usan redes sociales no desarrollan las habilidades de lectura, que no aprenden, que no tienen capacidad ni pensamiento crítico. En tanto que otros docentes si creen que el uso de redes sociales es de beneficio para los estudiantes. Esta dicotomía permitió sustentar la necesidad de un estudio que lleve a determinar si el uso de redes sociales resulta positivo para la formación de los estudiantes, y así plantear y recomendar acciones a los maestros y a la institución. A esta necesidad se suma también que, a nivel de Perú, no existen estudios que busquen determinar la influencia entre el uso de redes sociales, como espacio en el que de forma natural se realiza la lectura hipertextual, con las estrategias de lectura crítica hipertextual. En línea a lo anterior, el presente estudio determinará si el uso de redes sociales como tema principal influye significativamente en la lectura crítica hipertextual de los estudiantes de primer año de la Escuela de Economía de la UNFV, estas fundamentadas en la teoría.

\section{Método}

El presente estudio se encuentra dentro del paradigma positivista, el cual, según Rivas (2014), busca elaborar leyes científicas, dar explicaciones causales y predecir hechos tanto en lo social como en lo natural. El enfoque es de tipo cuantitativo, el nivel relacional y el diseño no experimental de tipo transversal. En lo referente a la población, esta estuvo conformada por 150 alumnos del primer año de la Escuela de Economía de la Universidad Nacional Federico Villarreal (UNFV), distribuidos en 3 aulas de 50 estudiantes cada una.

Para el recojo de información del uso de RRSS se utilizó un cuestionario cuyas escalas de frecuencia fueron de tipo cualitativa ordinal, con ítems que abarcan información sobre días y horas valorados según frecuencia de uso: nunca, casi nunca, a veces, casi siempre y siempre. Para las estrategias de lectura crítica hipertextual se utilizó el cuestionario de Galindo (2015), el cual cuenta con 4 dimensiones: la primera, estrategias de lectura hipertextual, con 13 ítems; la segunda, estándares intelectuales, con 9 ítems: la tercera, elementos de pensamiento, con 9 ítems; y la cuarta, comprobación del razonamiento crítico, con 9 items respectivamente. Todas las dimensiones se 
midieron en escala tipo likert con valores de 1 (pesimo), 2 (deficiente), 3 (regular), 4 (buena) y 5 (excelente). El proceso de validación fue por contenido realizado por expertos temáticos. Asimismo, se realizó una prueba piloto con el fin de obtener un índice de fiabilidad. Para esto se aplicó la prueba de alpha de Cronbach el cual arrojó un 0.801 para uso de redes sociales y 0.918 para el cuestionario de estrategias de lectura crítica hipertextual.

Para el procesamiento de la información se utilizó el paquete estadístico SPSS, v. 24. Se estableció los baremos para cada variable que permitió el análisis de los resultados descriptivos. De igual manera, para la prueba de la normalidad se empleó el estadístico Kolmogorov - Smirnov como prueba de decisión estadístico debido a que la muestra de estudios fue mayor a 30 sujetos. Los datos de ambas variables resultaron no normales es por ello que para la contratación de hipótesis se optó por un estadístico no paramétrico, en este caso, Rho de Spearman.

\section{Resultados}

En la tabla 1 se observa que, entre los datos más importantes, el $77.3 \%$ de los estudiantes refieren que ingresan todos los días al Facebook y un 53.3\%, que ingresa entre 2 y 3 horas diarias. Con respecto a Instagram, el $40.7 \%$ de los encuestados afirma no utilizar esta red social, mientras que el $44.7 \%$ indica que lo hace todos los días. El 50.7\% utiliza esta red menos de 2 horas diarias. Y con respecto al Twitter, el $92.7 \%$ afirma que no usa esta red social.

Tabla 1.

Resultados por ítem de uso redes sociales (RRSS) de los estudiantes del primer año de la Escuela de Economía de la UNFV

\begin{tabular}{|c|c|c|c|c|c|c|c|c|c|c|c|}
\hline \multirow{3}{*}{$\begin{array}{c}\text { Pregunta } \\
\text { 1. ¿Cuántos días } \\
\text { a la semana haces } \\
\text { uso Facebook? }\end{array}$} & \multicolumn{2}{|c|}{ Nunca } & \multicolumn{2}{|c|}{$\begin{array}{c}\text { Una vez a la } \\
\text { semana }\end{array}$} & \multicolumn{2}{|c|}{$\begin{array}{c}2 \text { días a la } \\
\text { semana }\end{array}$} & \multicolumn{2}{|c|}{$\begin{array}{c}\text { 3-4 días a la } \\
\text { semana }\end{array}$} & \multicolumn{2}{|c|}{$\begin{array}{c}\text { Todos los } \\
\text { días }\end{array}$} & \multirow[t]{2}{*}{ Total } \\
\hline & $\mathrm{f}$ & $\%$ & $\mathrm{f}$ & $\%$ & $\mathrm{f}$ & $\%$ & $\mathrm{f}$ & $\%$ & $\mathrm{f}$ & $\%$ & \\
\hline & 1 & 0.7 & 3 & 2.0 & 11 & 7.3 & 19 & 12.7 & 116 & 77.3 & 150 \\
\hline \multirow{3}{*}{$\begin{array}{c}\text { 2. ¿Cuántas horas } \\
\text { diarias ingresas } \\
\text { en promedio al } \\
\text { Facebook? }\end{array}$} & \multicolumn{2}{|c|}{$\begin{array}{c}\text { No } \\
\text { ingreso }\end{array}$} & \multicolumn{2}{|c|}{$\begin{array}{l}\text { Menos de } 2 \\
\text { horas }\end{array}$} & \multicolumn{2}{|c|}{$\begin{array}{c}\text { Entre } 2 \text { y } 3 \\
\text { horas }\end{array}$} & \multicolumn{2}{|c|}{$\begin{array}{l}\text { Entre } 3 \text { y } 5 \\
\text { horas }\end{array}$} & \multicolumn{2}{|c|}{$\begin{array}{c}\text { Más de } 5 \\
\text { horas }\end{array}$} & Total \\
\hline & $\mathrm{f}$ & $\%$ & F & $\%$ & $\mathrm{f}$ & $\%$ & $\mathrm{f}$ & $\%$ & $\mathrm{f}$ & $\%$ & \\
\hline & 2 & 3.0 & 42 & 28.0 & 80 & 53.3 & 12 & 8.0 & 13 & 8.7 & 150 \\
\hline \multirow{3}{*}{$\begin{array}{l}\text { 3. ¿Cuántos días } \\
\text { a la semana haces } \\
\text { uso Instagram? }\end{array}$} & \multicolumn{2}{|c|}{ Nunca } & \multicolumn{2}{|c|}{$\begin{array}{l}\text { Una vez a la } \\
\text { semana }\end{array}$} & \multicolumn{2}{|c|}{$\begin{array}{c}2 \text { días a la } \\
\text { semana }\end{array}$} & \multicolumn{2}{|c|}{$\begin{array}{c}\text { 3-4 días a la } \\
\text { semana }\end{array}$} & \multicolumn{2}{|c|}{$\begin{array}{l}\text { Todos los } \\
\text { días }\end{array}$} & Total \\
\hline & $\mathrm{f}$ & $\%$ & $\mathrm{f}$ & $\%$ & $\mathrm{f}$ & $\%$ & $\mathrm{f}$ & $\%$ & $\mathrm{f}$ & $\%$ & \\
\hline & 6 & 41 & 8 & 5.3 & 10 & 6.7 & 4 & 2.7 & 67 & 44.7 & 150 \\
\hline \multirow{3}{*}{$\begin{array}{l}\text { 4. ¿Cuántas horas } \\
\text { diarias ingresas } \\
\text { en promedio al } \\
\text { Instagram? }\end{array}$} & \multicolumn{2}{|c|}{$\begin{array}{c}\text { No } \\
\text { ingreso }\end{array}$} & \multicolumn{2}{|c|}{$\begin{array}{c}\text { Menos de } 2 \\
\text { horas }\end{array}$} & \multicolumn{2}{|c|}{$\begin{array}{c}\text { Entre } 2 \text { y } 3 \\
\text { horas }\end{array}$} & \multicolumn{2}{|c|}{$\begin{array}{c}\text { Entre } 3 \text { y } 5 \\
\text { horas }\end{array}$} & \multicolumn{2}{|c|}{$\begin{array}{c}\text { Más de } 5 \\
\text { horas }\end{array}$} & Total \\
\hline & $\mathrm{f}$ & $\%$ & $\mathrm{f}$ & $\%$ & $\mathrm{f}$ & $\%$ & $\mathrm{f}$ & $\%$ & $\mathrm{f}$ & $\%$ & \\
\hline & & 41 & 76 & 51 & 8 & 5.3 & 3 & 2.0 & 1 & 0.7 & 150 \\
\hline \multirow{3}{*}{$\begin{array}{l}\text { 5. ¿Cuántos días } \\
\text { a la semana haces } \\
\text { uso de Twitter? }\end{array}$} & \multicolumn{2}{|c|}{ Nunca } & \multicolumn{2}{|c|}{$\begin{array}{c}\text { Una vez a la } \\
\text { semana }\end{array}$} & \multicolumn{2}{|c|}{$\begin{array}{c}2 \text { días a la } \\
\text { semana }\end{array}$} & \multicolumn{2}{|c|}{$\begin{array}{c}\text { 3-4 días a la } \\
\text { semana }\end{array}$} & \multicolumn{2}{|c|}{$\begin{array}{l}\text { Todos los } \\
\text { días }\end{array}$} & Total \\
\hline & $\mathrm{f}$ & $\%$ & $\mathrm{f}$ & $\%$ & $\mathrm{f}$ & $\%$ & $\mathrm{f}$ & $\%$ & $\mathrm{f}$ & $\%$ & \\
\hline & 136 & 91.0 & 6 & 4.0 & 4 & 2.7 & 1 & 0.7 & 3 & 2.0 & 150 \\
\hline
\end{tabular}




\begin{tabular}{|c|c|c|c|c|c|c|c|c|c|c|c|}
\hline \multirow{3}{*}{$\begin{array}{c}\text { 6. ¿Cuántas horas } \\
\text { diarias ingresas } \\
\text { en promedio al } \\
\text { Twitter? }\end{array}$} & \multicolumn{2}{|c|}{$\begin{array}{c}\text { No } \\
\text { ingreso }\end{array}$} & \multicolumn{2}{|c|}{$\begin{array}{c}\text { Menos de } 2 \\
\text { horas }\end{array}$} & \multicolumn{2}{|c|}{$\begin{array}{c}\text { Entre } 2 \text { y } 3 \\
\text { horas }\end{array}$} & \multicolumn{2}{|c|}{$\begin{array}{c}\text { Entre } 3 \text { y } 5 \\
\text { horas }\end{array}$} & \multicolumn{2}{|c|}{$\begin{array}{c}\text { Más de } 5 \\
\text { horas }\end{array}$} & \multirow[t]{2}{*}{ Total } \\
\hline & $\mathrm{f}$ & $\%$ & $\mathrm{f}$ & $\%$ & $\mathrm{f}$ & $\%$ & $\mathrm{f}$ & $\%$ & $\mathrm{f}$ & $\%$ & \\
\hline & 138 & 92.0 & 8 & 5.3 & 0 & 0.0 & 4 & 2.7 & 0 & 0.0 & 150 \\
\hline \multirow[t]{3}{*}{$\begin{array}{l}\text { 7. ¿Cuántos días } \\
\text { a la semana haces } \\
\text { uso de Youtube? }\end{array}$} & \multicolumn{2}{|c|}{ Nunca } & \multicolumn{2}{|c|}{$\begin{array}{c}\text { Una vez a la } \\
\text { semana }\end{array}$} & \multicolumn{2}{|c|}{$\begin{array}{c}2 \text { días a la } \\
\text { semana }\end{array}$} & \multicolumn{2}{|c|}{$\begin{array}{c}\text { 3-4 días a la } \\
\text { semana }\end{array}$} & \multicolumn{2}{|c|}{$\begin{array}{l}\text { Todos los } \\
\text { días }\end{array}$} & Total \\
\hline & $\mathrm{f}$ & $\%$ & $\mathrm{f}$ & $\%$ & $\mathrm{f}$ & $\%$ & $\mathrm{f}$ & $\%$ & $\mathrm{f}$ & $\%$ & \\
\hline & 3 & 2 & 7 & 4.7 & 10 & 6.7 & 85 & 56.7 & 45 & 30 & 150 \\
\hline \multirow{3}{*}{$\begin{array}{c}\text { 8. ¿Cuántas horas } \\
\text { diarias ingresas } \\
\text { en promedio al } \\
\text { Youtube? }\end{array}$} & \multicolumn{2}{|c|}{$\begin{array}{c}\text { No } \\
\text { ingreso }\end{array}$} & \multicolumn{2}{|c|}{$\begin{array}{c}\text { Menos de } 2 \\
\text { horas }\end{array}$} & \multicolumn{2}{|c|}{$\begin{array}{c}\text { Entre } 2 \text { y } 3 \\
\text { horas }\end{array}$} & \multicolumn{2}{|c|}{$\begin{array}{c}\text { Entre } 3 \text { y } 5 \\
\text { horas }\end{array}$} & \multicolumn{2}{|c|}{$\begin{array}{c}\text { Más de } 5 \\
\text { horas }\end{array}$} & Total \\
\hline & $\mathrm{f}$ & $\%$ & $\mathrm{f}$ & $\%$ & $\mathrm{f}$ & $\%$ & $\mathrm{f}$ & $\%$ & $\mathrm{f}$ & $\%$ & \\
\hline & 3 & 2 & 34 & 23 & 80 & 53.3 & 22 & 14.7 & 11 & 7.3 & 150 \\
\hline
\end{tabular}

En la figura 1 se aprecia que el $1.3 \%$ de los estudiantes encuestados usan de manera regular la estrategia de lectura hipertextual y nunca usan las redes sociales, mientras el $35.3 \%$ de los estudiantes considera que tiene buenas estrategias de lectura Hipertextual y al mismo tiempo, usan a veces las redes sociales (RRSS). Por lo identificado podemos afirmar que los estudiantes que usan redes sociales tienen estrategias de lectura hipertextual.

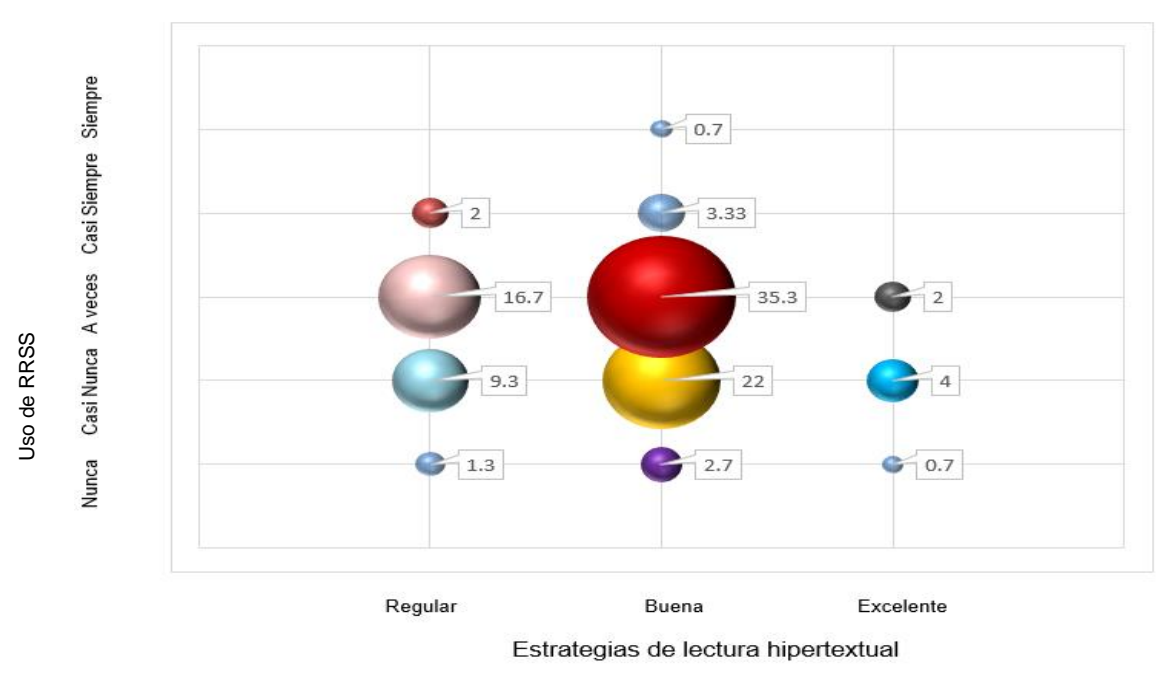

Figura 1. Uso de redes sociales (RRSS) y estrategias de lectura hipertextual de los estudiantes del primer año de la Escuela de Economía de la UNFV.

En la figura 2 se aprecia que el 1.3\% de los estudiantes encuestados consideran se encuentran en un nivel regular respecto a la comprobación del razonamiento crítico y que nunca usan redes sociales; mientras el 34\% de los estudiantes considera que tiene una buena comprobación del razonamiento crítico y que a veces usan redes sociales. Podemos afirmar que los estudiantes que usan redes sociales practican la comprobación del razonamiento crítico. 


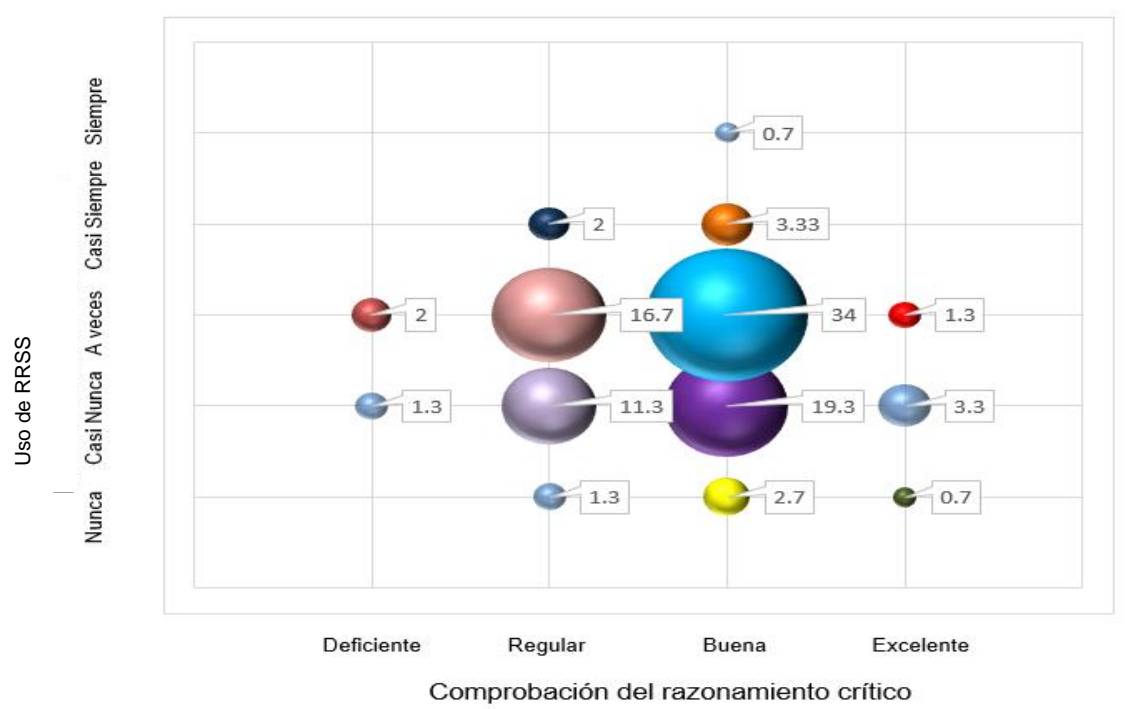

Figura 2. Uso de redes sociales (RRSS) y comprobación del razonamiento crítico de los estudiantes del primer año de la Escuela de Economía de la UNFV.

De la figura 3, se aprecia que el $1.3 \%$ de los estudiantes encuestados presentan en nivel regular elementos de pensamiento, y que nunca usan redes sociales; mientras que el $35.3 \%$ de los estudiantes considera que tiene buenos elementos de pensamiento y al mismo tiempo, considera que a veces usan las redes sociales (RRSS). Podemos afirmar que un elemento importante para desarrollar los elementos de pensamiento es el uso de redes sociales

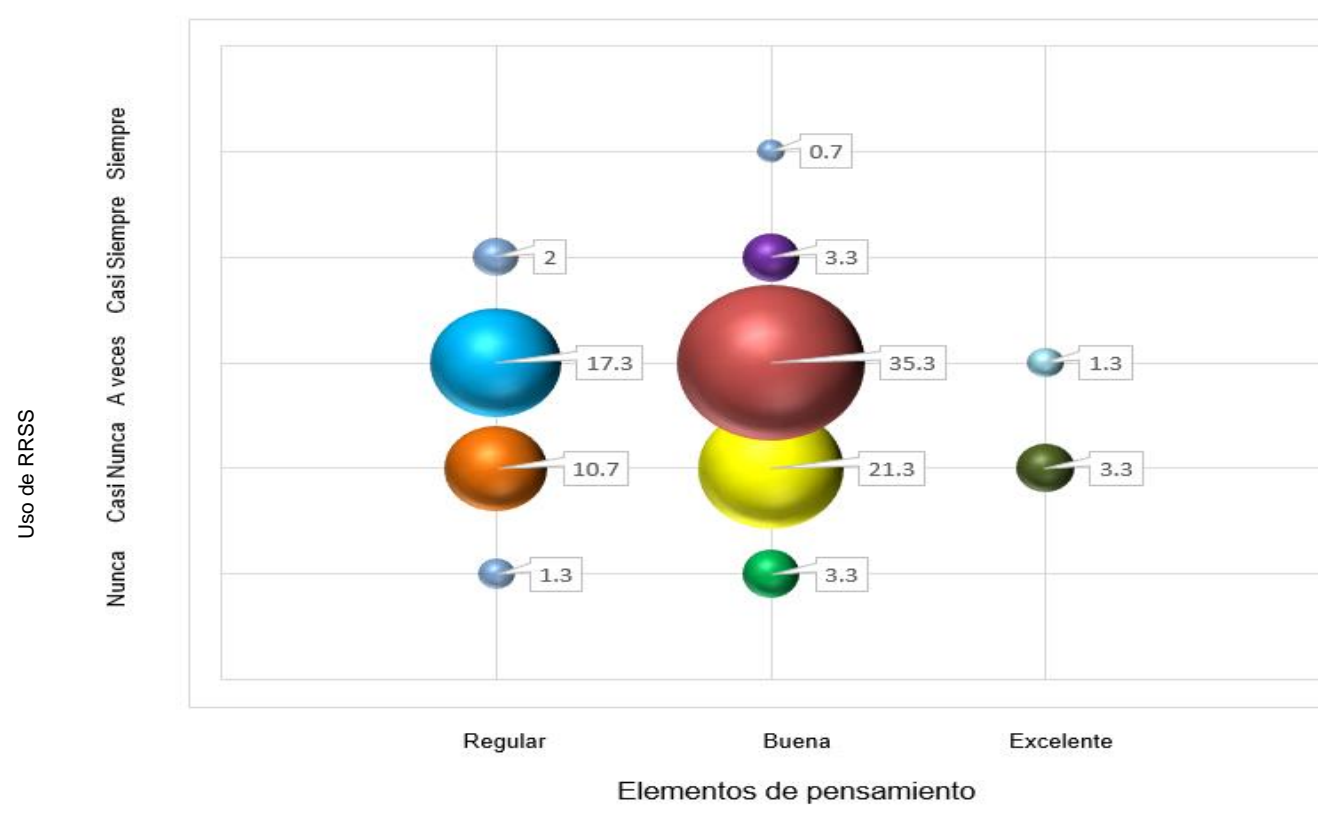

Figura 3. Uso de redes sociales (RRSS) y elementos del pensamiento de los estudiantes del primer año de la Escuela de Economía de la UNFV.

\section{Discusión}

Los estudiantes actuales están en constante contacto con redes sociales y otras herramientas de carácter hipertextual. A lo largo de su formación van creando formas distintas de leer y de procesar la información con el propósito de comprender lo que se transmite y así poder seleccionar la 
información. En vista de esta realidad, se hace necesario saber si estos hábitos están asociados con estrategias críticas para la lectura de hipertextos que coadyuven a seleccionar y dar valor a los distintos materiales que ellos utilizarán para desarrollar sus aprendizajes, así como para la elaboración de trabajos en su formación profesional, teniendo en cuenta de que existe diversa información en internet. Los resultados obtenidos en el presente estudio resultan similares a los hallados por Almansa, Fonseca y Castillo (2013) quienes, bajo un enfoque cualitativo, aplicaron entrevistas de carácter comparativo tanto en el país de Colombia como en España. Entre sus hallazgos figuran que, en estos países, gran parte de los adolescentes de 12 a 15 años utilizan la plataforma FB para interactuar con sus amigos y amigas, siendo así el medio de mayor socialización. Asimismo, exponen un lenguaje de comunicación propia, que son totalmente ajenas a toda norma ortográfica y gramática.

Por el lado peruano, GFK Perú (2015) realizó una investigación sobre el uso del Internet en el Perú, en donde se puede observar que, con respecto al uso de redes sociales, el $90 \%$ de peruanos usa Facebook; el 55\%, YouTube; el 53\%, Google; y 17\%, Twitter, teniendo la misma línea los resultados que se obtuvieron en el presente trabajo de investigación. En lo que se refiere al uso de las estrategias de lectura crítica hipertextual, los alumnos del primer año de la Facultad de Economía de la UNFV refieren que el 34\% de los alumnos hacen uso a un nivel regular de las estrategias; el 62\% buena y el $4 \%$ excelente uso de las estrategias. Resultados similares fueron los obtenidos por Galindo (2015) de la Universidad de la Sonora, quien halló que los estudiantes universitarios utilizan estrategias críticas hipertextuales con un comportamiento favorable, un poco regular y bueno, y que en cuanto a aspectos específicos de las estrategias de la lectura hipertextual como son el análisis y la evaluación, demuestran mejores puntuaciones frente a habilidades de solo recolección de datos.

Con respecto a la relación entre ambas variables y cada uno de sus componentes, se aceptaron las hipótesis nulas, es decir, no hay relación significativa entre el uso redes sociales (RRSS) y el nivel de uso de estrategias de lectura crítica hipertextual y sus dimensiones: elementos de pensamiento, estrategias de lectura hipertextual, comprobación del pensamiento crítico y estándares intelectuales. En el caso peruano, Ruiz y Escurra (2013) realizaron un estudio con resultados cercanos a los hallados en el presente estudio, sobre la relación que se da entre el uso del FB (Facebook) y YouTu (YouTube), con la conciencia metacognitiva, las estrategias metacognitivas en la lectura y aprendizaje. El resultado de este último estudio mostró un mayor uso del FB (Facebook), pero bajos niveles en el desarrollo de la conciencia metacognitiva, así como bajo uso de estrategias metacognitivas en la lectura. Dicho estudio brinda también una relación significativa, negativa y moderada entre los hábitos de consumo de las plataformas en línea y la conciencia metacognitiva; de igual manera, muestra una relación significativa negativa y baja entre los hábitos de consumo de las plataformas en línea, las estrategias metacognitivas en la lectura y las estrategias de aprendizaje y estudio.

Por lo antes expuesto los estudiantes viven en una cultura digital compartiendo información, interactuando en una biblioteca, el cual debe estar conectada y facilitar el acceso a una amplia red de información de formatos hipertextuales, en un espacio donde se promueva la comunicación multimedia y audio-visual. La mayor cantidad de interacción, así como el consumo de información, se da principalmente mediante la lectura hipertextual y multimedia, por ello, para los estudiantes, se ha vuelto necesario manejar adecuadamente la lectura hipertextual desde una perspectiva crítica y reflexiva.

\section{Referencias}

Almansa, A., Fonseca, O., \& Castillo, A. (2013). Redes sociales y jóvenes. Uso de Facebook en la juventud colombiana y española. Comunicar, 40(20), 127-135. doi: https://doi.org/10.3916/C40-2013-03-03

Arancibia, M.N. (2010). Estrategias de comprensión con hipertexto informativo. Recuperado de http://www.lecturayvida.fahce.unlp.edu.ar/numeros/a31n2/31_02_Arancibia.pdf 
Eco, U. (2003). Vegetal and mineral memory. The future of books, Discurso de inauguración de la Biblioteca de Alejandría. En Mendoza, A. (coord.) Leer hipertextos. Del marco hipertextual a la formación del lector literario (pp.9-32). Barcelona: Octaedro

Galindo, M.A. (2015). Lectura crítica hipertextual en la web 2.0. Actualidades investigativas en educación, 15(1), 1-29. Recuperado de https://revistas.ucr.ac.cr/index.php/aie/article/view/16972

GFK. (2015). Uso de internet en el Perú. Recuperado de https://www.gfk.com/fileadmin/user_upload/dyna_content/PE/GfK_OP_Octubre_2015__Uso_de_Internet_2.pdf.

Instituto Nacional de Tecnologías de la Comunicación (2009). Estudio sobre la privacidad de los datos y la seguridad de la información en las redes sociales online.

Mendoza, A. (2012). Leer hipertextos. Del marco hipertextual a la formación del lector literario. Barcelona: Octaedro.

Orellana, L. M. (2012). Uso de internet por jóvenes universitarios de la Facultad de Ciencias Sociales de la Universidad Nacional Federico Villarreal. (Tesis de Maestría. Universidad Nacional Mayor de San Marcos). Lima, Perú.

Rivas, F. (2014). Diccionario de investigación científica cuantitativa y cualitativa. Lima: Concytec. Romea,

Romea, C. (2012). Cleopatra, un nodo hipermediático con más de 2000 años. En Antonio Mendoza (coord.), Leer hipertextos. Del marco hipertextual a la formación del lector literario (pp. 246-261). España: Octaedro.

Ruiz, F., \& Escurra, L. M. (2013). Hábitos de consumo de Facebook y Youtube. Conciencia y estrategias metacognitivas en la lectura y estrategias de aprendizaje y estudio en universitarios. Persona, 16, 29-71. doi: https://doi.org/10.26439/persona2013.n016.2

San Martín, L., \& Mujica, S. (2010). Redes sociales: la nueva forma de comunicación de los jóvenes universitarios. 8th Latin American and Caribbean Conference for Engineering and Technology.

Schwarz, B. (2011). Spouses' Demand and Withdrawal During Marital Conflict in Relation to Their Subjective Well-Being. Journal of Social and Personal Relationships, 28, 262-277. doi: https://doi.org/10.1177/0265407510382061

Siemens, G. (2006). Connectivism: Learning Theory or Pastime for the Self-Amused? Recuperado de https://altamirano.biz/conectivismo.pdf. 